\title{
Organisation de sessions de formation continue: un regard sur les réglementations à l'étranger
}

\author{
Annina Vogler ${ }^{a}$, \\ Hermann Amstad ${ }^{b}$ \\ a MLaw, Collaboratrice \\ scientifique à l'Institut für \\ Völkerrecht, Université de \\ Zurich \\ b Dr, secrétaire général de \\ l'ASSM, Bâle
}

La confiance de la société envers la profession médicale repose sur la crédibilité du corps médical. Pour légitimer cette confiance, une gestion transparente des conflits d'intérêts, c'est-à-dire des intérêts divergents primaires et secondaires [1], est indispensable. Et l'absence de transparence mettrait justement en péril ces qualités inhérentes à la profession.

En Suisse, la formation continue à vie est une obligation pour les professionnels de la santé; pourtant il n'existe aucune disposition légale qui en fixe les principales conditions cadres - notamment en matière de conflits d'intérêts. Consciente de cette situation, l'Académie Suisse des Sciences Médicales (ASSM) a publié en 2006 des directives concernant la «Collaboration corps médical-industrie» [2], par leur intégration au code de déontologie de la FMH, celles-ci sont devenues contraignantes [3] pour le corps médical.

Or, ces directives font constamment l'objet de discussions. D'aucuns les trouvent trop sévères ou difficiles à mettre en pratique. Face à ce constat, la Commission Consultative de l'ASSM (chargée de veiller à la mise en œuvre des directives) a décidé d'étudier les législations correspondantes au niveau international resp. dans d'autres pays. Après avoir entrepris des recherches détaillées sur Internet, elle a adressé, fin mai 2010, un questionnaire concernant les réglementations en vigueur dans leurs pays à 27 sociétés médicales nationales en Europe et en Amérique du Nord. Après un courrier de rappel, les sociétés médicales de 10 pays avaient répondu fin juillet (voir tableau 1).

\section{Réglementations internationales}

L'Organisation Mondiale de la Santé (OMS) avait révisé en 2009 le «Statement concerning the relationship between physicians and commercial enterprises». Toutefois, le document révisé est resté aussi modeste dans ses exigences que la version de 2004 . Les principaux points révisés concernent la transparence et l'indépendance relative au contenu; par contre, le règlement ne s'exprime pas sur le monosponsoring et l'utilisation de notions juridiques imprécises [4] lui enlève également de son efficacité. Même si les directives de l'OMS n'engagent pas juridiquement, elles ne jouent pas moins un rôle de précurseur dans les règlementations internationales. Il n'existe donc aucun moyen pour vérifier si les différents membres ont déjà mis en œuvre les directives. Dans la plupart des pays, les associations de médecins sont responsables de l'élaboration et de la mise en œuvre des normes éthiques en vigueur. En fonction de la position du pays concerné face au droit médical, ces normes peuvent avoir force de loi. Toujours est-il que ces directives de l'OMS peuvent combler une lacune dans des pays qui ne disposent pas de législation dans ce domaine.

\section{Comparaison des réglementations nationales}

Tous les pays qui ont répondu à l'enquête ne prévoient pas une obligation de formation continue, comme c'est le cas en Suisse [5]. Différentes organisations peuvent proposer des sessions de formation pour les médecins: hôpitaux, sociétés de discipline médicale, associations régionales ou nationales de médecins, entreprises pharmaceutiques ou instituts de formation privés. En conséquence, les réponses présentaient une grande diversité. Il convient de souligner que ce n'est qu'en Allemagne et au Danemark que des entreprises pharmaceutiques peuvent organiser des sessions de formation.

Dans chaque pays, la collaboration entre le corps médical et l'industrie, dans le domaine de la formation continue et postgraduée, fait l'objet de réglementations - indépendamment du degré de norme. En revanche, le monosponsoring d'une session de formation continue par une entreprise privée n'est interdit qu'en Allemagne, au Portugal et en République tchèque. On peut souligner à cet égard que ni la Finlande, ni les Etats-Unis ne disposent de règlementation à ce sujet.

\section{Les directives suisses sont conformes au développement international}

Dans tous les pays, à l'exception du Danemark et de la Hongrie, les conflits d'intérêts doivent être révélés, qu'ils soient personnels ou institutionnels [6]. Les réponses concernant la participation aux frais d'une session de formation pour les médecins participants sont nettement moins homogènes: Pour la moitié des organisations sondées, des droits d'inscription adéquats sont la règle; parmi les pays sondés, trois d'entre eux ne disposent d'aucun règlement à ce sujet et deux pays n'ont donné aucune indication. L'exigence d'une convention écrite entre les organisateurs et les sponsors fait, elle aussi, l'objet de divergences: La Finlande et l'Autriche ne demandent de conventions écrites ni pour ce qui concerne les détails de la 
prestation à soutenir, ni même pour la comptabilisation des fonds sur un compte de l'organisateur ouvert à cet effet. L'Allemagne, la Norvège et le Portugal n'ont donné aucune information à ce sujet. Les autres organisations sondées prévoient explicitement d'introduire de telles directives. L'introduction d'une réglementation concernant l'indépendance de l'organisateur quant au contenu, au libre choix des confé- renciers et au déroulement de la manifestation recueille l'unanimité. Il en va de même pour la réglementation du programme cadre en marge de la manifestation, qui doit revêtir une importance secondaire par rapport à la partie professionnelle.

L'association américaine des médecins a publié une «Ethical Opinion» concernant les cadeaux faits par l'industrie aux médecins [7]. Ce document inter-

Tableau 1

Réponses de 10 pays.

\begin{tabular}{|c|c|c|c|c|c|c|c|c|c|c|}
\hline & 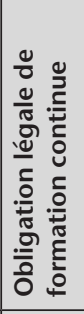 & 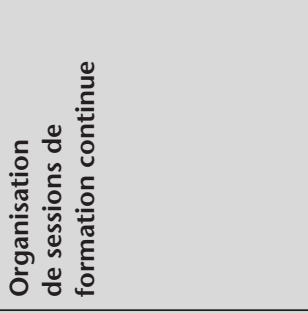 & 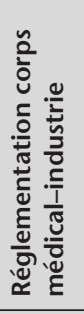 & 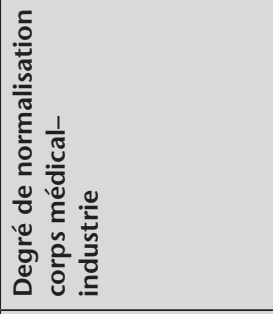 & 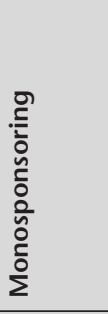 & 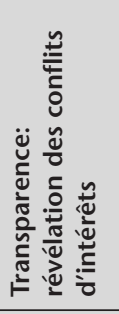 & 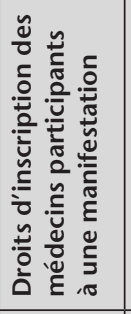 & 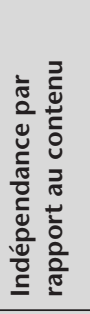 & 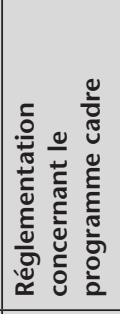 & 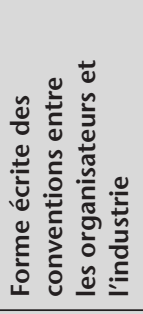 \\
\hline Danemark & non & $\begin{array}{l}\text { Hôpitaux, sociétés de dis- } \\
\text { cipline médicale, } \\
\text { associations de médecins, } \\
\text { entreprises pharma- } \\
\text { ceutiques, instituts de } \\
\text { formation privés }\end{array}$ & oui & $\begin{array}{l}\text { Code de déontologie } \\
\text { médicale, directives } \\
\text { spécifiques }\end{array}$ & possible & non & $\begin{array}{l}\text { aucun } \\
\text { règle- } \\
\text { ment }\end{array}$ & oui & oui & oui \\
\hline Allemagne & oui & $\begin{array}{l}\text { Hôpitaux, sociétés de dis- } \\
\text { cipline médicale, } \\
\text { associations de médecins, } \\
\text { entreprises pharma- } \\
\text { ceutiques, instituts de } \\
\text { formation privés }\end{array}$ & oui & $\begin{array}{l}\text { Code de déontologie } \\
\text { médicale, directives } \\
\text { spécifiques }\end{array}$ & interdit & oui & $\begin{array}{l}\text { aucun } \\
\text { règle- } \\
\text { ment }\end{array}$ & oui & oui & $\begin{array}{l}\text { pas d'in- } \\
\text { dications }\end{array}$ \\
\hline Finlande & non & $\begin{array}{l}\text { Hôpitaux, sociétés de dis- } \\
\text { cipline médicale }\end{array}$ & oui & $\begin{array}{l}\text { Code de déontologie } \\
\text { médicale, directives } \\
\text { spécifiques }\end{array}$ & possible & oui & oui & oui & oui & non \\
\hline Norvège & non & $\begin{array}{l}\text { Hôpitaux, sociétés de dis- } \\
\text { cipline médicale, } \\
\text { associations de médecins, } \\
\text { facultés de médecine }\end{array}$ & oui & Directives spécifiques & $\begin{array}{l}\text { aucun } \\
\text { règle- } \\
\text { ment }\end{array}$ & oui & \begin{tabular}{|l|} 
pas d'in- \\
dications
\end{tabular} & oui & oui & $\begin{array}{l}\text { pas d'in- } \\
\text { dications }\end{array}$ \\
\hline Autriche & oui & $\begin{array}{l}\text { Hôpitaux, sociétés de dis- } \\
\text { cipline médicale, } \\
\text { associations de médecins, } \\
\text { instituts de formation } \\
\text { privés, facultés de } \\
\text { médecine, autres } \\
\text { fournisseurs du domaine } \\
\text { médical }\end{array}$ & oui & $\begin{array}{l}\text { Code de déontologie } \\
\text { médicale, directives } \\
\text { spécifiques }\end{array}$ & possible & oui & oui & oui & oui & non \\
\hline Portugal & non & $\begin{array}{l}\text { Hôpitaux, sociétés de dis- } \\
\text { cipline médicale }\end{array}$ & oui & $\begin{array}{l}\text { Code de déontologie } \\
\text { médicale, directives } \\
\text { spécifiques }\end{array}$ & interdit & $\begin{array}{l}\text { pas d'in- } \\
\text { dications }\end{array}$ & $\begin{array}{l}\text { pas d'in- } \\
\text { dications }\end{array}$ & oui & oui & $\begin{array}{l}\text { pas d'in- } \\
\text { dications }\end{array}$ \\
\hline $\begin{array}{l}\text { République } \\
\text { tchèque }\end{array}$ & oui & Hôpitaux & oui & Directives spécifiques & interdit & oui & oui & oui & oui & oui \\
\hline Hongrie & oui & $\begin{array}{l}\text { Hôpitaux, sociétés de dis- } \\
\text { cipline médicale, } \\
\text { associations de médecins }\end{array}$ & oui & Directives spécifiques & possible & non & oui & oui & oui & oui \\
\hline Canada & oui & $\begin{array}{l}\text { Hôpitaux, sociétés de dis- } \\
\text { cipline médicale, } \\
\text { associations de médecins, } \\
\text { instituts de formation } \\
\text { privés }\end{array}$ & oui & Directives spécifiques & possible & oui & oui & oui & oui & oui \\
\hline Etats-Unis & oui & pas d'indications & oui & Directives spécifiques & $\begin{array}{l}\text { aucun } \\
\text { règle- } \\
\text { ment }\end{array}$ & oui & $\begin{array}{l}\text { aucun } \\
\text { règle- } \\
\text { ment }\end{array}$ & oui & oui & oui \\
\hline
\end{tabular}


dit explicitement aux entreprises pharmaceutiques de prendre en charge les éventuels frais de déplacement et autres frais (repas, nuitées, etc.) occasionnés par la participation à des sessions de formation. L'association médicale du Canada proscrit également de telles indemnisations. Par ailleurs, la remise de cadeaux personnels «de valeur élevée» est interdite; mais contrairement à la directive américaine [8], la réglementation canadienne ne donne aucune indication quant à la valeur ni quant à l'affectation. La possibilité de perdre son autonomie est cependant mentionnée explicitement [9].

\section{Conclusion}

Dans la comparaison internationale, les directives de l'ASSM font bel et bien partie des réglementations les plus sévères. S'il est vrai qu'elles ne contiennent aucune exigence qui n'existerait nulle part ailleurs, nos recherches ne nous ont pas non plus permis de trouver des dispositions qui ne seraient ni connues ni appliquées en Suisse.

Si l'on consulte la littérature internationale sur ces thèmes (cf. à titre d'exemple l'éditorial de Robert F. Woollard: "Continuing medical education in the 21st century» [10]; en français dans le Bulletin des médecins suisses [11]), on constate que les directives suisses sont conformes au développement international resp. que ces exigences sont déjà largement entrées en vigueur. Pour une fois, la Suisse fait donc partie de l'avant-garde.
1 C'est-à-dire des intérêts des patients resp. des intérêts commerciaux.

2 Ces directives peuvent être consultées sous: www.samw.ch/fr/Ethique/Corps-medical-Industrie. html, dernière consultation le 3 septembre 2010.

3 Une infraction à ces directives par un représentant de la profession sera sanctionnée au moins au sein de la profession. Selon l'art. 43 du code de déontologie de la FMH, «Le code de déontologie engage tous les membres de la FMH, sauf dans les cas où le droit sanitaire cantonal prévoit des dispositions contraires».

4 Cf. Medical Conferences: «Physicians may not accept unjustified hospitality», www.wma.net/ en/30publications/10policies/r2/index.html, dernière consultation le 3 septembre 2010.

5 Ceci n'exclut évidemment pas une réglementation dans le code de déontologie.

6 Il manque des indications pour une solution à ce problème au Portugal.

7 Cf. Opinion E-8.061, Gifts to Physicians from Industries.

8 La directive américaine précise que seuls des cadeaux dont la valeur est inférieure ou égale à 100 USD et des «gifts that are primarly for the benefit of patients» peuvent être acceptés.

9 CMA Policy, Guidelines for Physicians in Interaction with Industry.13.

10 Woollard RF. Continuing medical education in the 21st century. British Medical Journal. 2008;337:a119.

11 Woollard RF. La formation continue des médecins au XXIe siècle. Bull Méd Suisses. 2009; 90(47):1828-30.

\section{Vous qui lisez une revue des Editions médicales suisses,}

\section{saviez-vous que ...}

- les Editions médicales suisses sont une coopération entre la Fédération des médecins suisses (FMH) et la plus ancienne maison d'édition et imprimerie au monde (les Editions Schwabe, fondées en 1488)?

- que les EMH sont les éditions de pointe en Suisse dans le domaine des journaux médicaux, avec dix revues spécialisées, des articles paraissant intégralement en ligne et un choix de livre de plus en plus large?

- que toutes les revues paraissant aux EMH sont les organes officiels de publication des sociétés médicales correspondantes?

Si vous souhaitez en savoir plus sur les EMH, vous trouverez plus d'informations sous www.emh.ch.

EMH Editions médicales suisses - des publications à la pointe de la médecine 